\title{
Lessons learnt in transitioning from universal screening to universal testing of pregnant patients for SARS-CoV-2 at the largest municipal health system in America
}

\author{
Wendy Wilcox $\mathbb{D}^{1} \cdot{\text { Komal Bajaj } \mathbb{D}^{2} \cdot \operatorname{Max} \text { C. Rossberg }}^{3} \cdot$ Clementine Knight $^{3} \cdot{\text { Daryl Wieland } \mathbb{D}^{2} \cdot \text { Yogangi Malhotra }}^{2}$
}

Received: 27 May 2020 / Revised: 22 September 2020 / Accepted: 20 November 2020 / Published online: 18 January 2021

(c) Springer Nature America, Inc. 2020

\section{Introduction}

As cases of coronavirus disease (COVID-19) surged worldwide, the guidelines around management of pregnant women admitted for delivery evolved quickly. Early in the pandemic, emphasis was placed on screening for travel history and symptoms. Based on CDC guidance, screening for symptoms was widely recommended as a way to approach pregnant patients [1]. By mid to late March, the significance of knowing the COVID-19 status of a patient was recognized in order to inform isolation precautions, allow for judicious use of PPE by hospital staff, and to guide postpartum management of both the mother and newborn [2]. On April 13, 2020, Sutton et al. highlighted the fact that $15 \%$ of women in their population in New York City were positive for severe acute respiratory syndrome coronavirus 2 (SARS-CoV-2) and of these, 90\% were asymptomatic at presentation [3]. It was clear that screening for symptoms and travel history were not clinically as informative as universal testing all pregnant patients for COVID-19 positivity using the reverse transcriptase polymerase chain reaction (rt-PCR).

Though there have been several studies comparing the prevalence of SARS-CoV-2 detected before and after universal testing of pregnant patients $[4,5]$, there is not a singular definition for "universal testing." There is variability in how different groups have reported results and the type of testing hasn't always been described $[3,6,7]$.

\footnotetext{
Wendy Wilcox

wendy.wilcox@nychhc.org

NYC Health + Hospitals/Kings County, Brooklyn, NY, USA

2 NYC Health + Hospitals/Jacobi, Bronx, NY, USA

3 Albert Einstein College of Medicine, Bronx, NY, USA
}

New York City Health + Hospitals $(\mathrm{NYC} \mathrm{H}+\mathrm{H})$, the largest municipal healthcare system in the United States (U.S.) of America delivers 16,000 pregnancies across 11 acute care facilities annually. It serves a demographic, which is uniquely different population than the one studied by Sutton et al., and is at the highest risk for infection [8]. Over $30 \%$ of our patients are uninsured, and over $60 \%$ self-identify as either Black/African American, Hispanic/Latino, or Asian [8]. The SARS-CoV-2-positive pregnant patients comprised 6\% white, $47.6 \%$ Black/African American, $19.7 \%$ Hispanic/ Latino, $5.7 \%$ Asian, and $40.8 \%$ other race/ethnicities.

This study provides a clear and specific definition of universal testing of pregnant patients presenting for their delivery. It also describes how testing was scaled up across 11 different departments of obstetrics within $\mathrm{NYC} \mathrm{H}+\mathrm{H}$ in order to respond to rapidly changing guidelines, testing methodologies and results availability. The power of systems was harnessed to promote best practices and facilitate adoption across otherwise independent departments of obstetrics.

\section{Materials and methods}

This study received IRB approval from the Biomedical Research Alliance of New York. A retrospective review of the electronic health records for women who delivered at the all of the $11 \mathrm{NYC} \mathrm{H}+\mathrm{H}$ facilities from March 1 to May 2, 2020 was conducted. Real-time weekly trends and variability in uptake in universal testing of patients were tracked in response to the implementation efforts across the system. Two rt-PCR tests were available- the Bioreference rt-PCR with a turnaround of 24-96h (BioReference Laboratories Inc, Spring Valley, NY, USA), and the Cepheid rapid rt-PCR (Cepheid, Sunnyvale, CA, USA) with a turnaround time of $1-2 \mathrm{~h}[9,10]$. Both were approved under Emergency Use Authorization by the United States Food 
Fig. 1 Trend of SARS-CoV-2 rt-PCR universal testing of delivered mothers at $\mathrm{NYC} \mathrm{H}+\mathrm{H}$ between weeks of Mar 1st and May 3rd 2020.

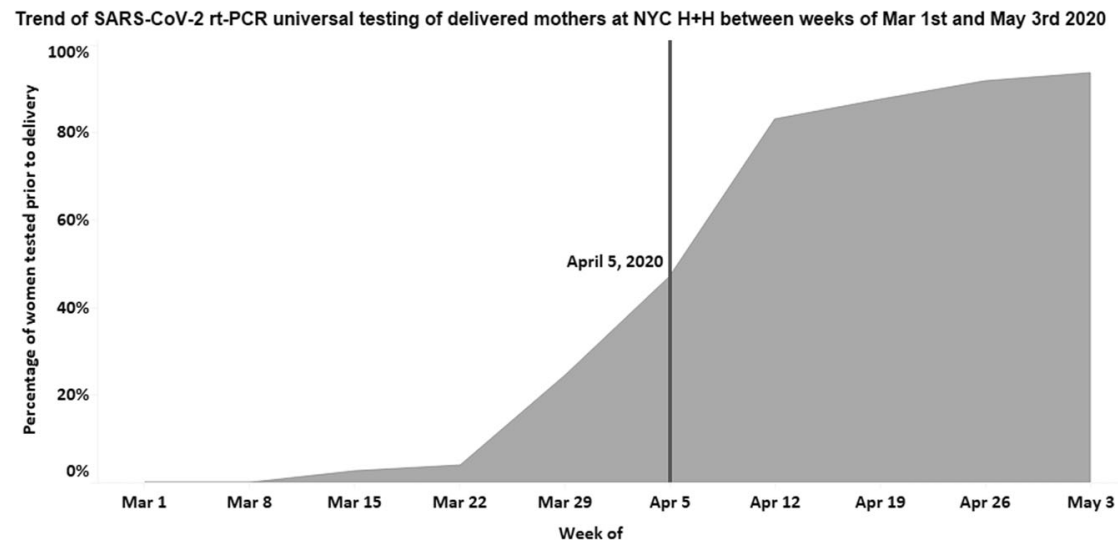

and Drug Administration. A nasopharyngeal swab was obtained for each patient and tested for SARS-CoV-2 using rt-PCR. The limit of detection (LOD) for the Bioreference rt-PCR is 25 genome equivalents/ml (100\% agreement at $1.5 \times$ LOD) while for the Cepheid rt-PCR test is 250 genome equivalents/ml, (100\% agreement at $2 \times$ LOD) [9, 10]. Further sensitivity and specificity analyses are unavailable at this time.

\section{Results}

From March 1 to April 4, 2020, a total of 75/1320 (6.1\%) women were tested for symptoms consistent with COVID19 before their delivery. Of these, $90.1 \%$ were tested using the BioReference rt-PCR. From April 5, 2020 to May 2, 2020 , the period of system-wide implementation, a total of $794 / 1025(77 \%)$ were tested prior to their delivery. Of these, $30 \%$ were using the BioReference PCR test, the majority being switched to the Cepheid rapid PCR test. By April 25, 87.6\% of the pregnant women received SARSCoV-2 testing regardless of symptoms or presentation (Fig. 1). The prevalence of SARS-CoV-2-positive patients in our population peaked at $27.1 \%$ during the week of April 12,2020 , while overall prevalence after uptake of universal testing 22.6\% between April 5 and May 2, 2020.

\section{Discussion}

We define "universal testing" as rt-PCR testing being done prior to the delivery of the infant during or prior to the admission of the patient. Studies that have reported universal testing as rt-PCR obtained prior to scheduled deliveries only, during the hospitalization but unclear whether the testing was done prior to the delivery of the infant cannot be considered universal testing $[3,6,7]$. The purpose of the universal testing is to ascertain prevalence of SARSCoV-2 among pregnant women and to guide management of the mother-baby dyad while allocating appropriate precautions and resources. The timing of the testing is critically important to inform the management of the dyad and to allocate resources.

There are barriers to successful universal testing of $100 \%$ of pregnant patients who are admitted for their delivery. As the health systems face the surge of critically ill patients, all resources, including testing supplies need judicious use. The other system and patient-based barriers range from timing of delivery, stage of labor at presentation, and the type of testing available, to the technical process and workflow involved in timely reporting of test results. Some patients refused testing for SARS-CoV-2.

The process of transitioning 11 labor and delivery units to one system of testing posed many challenges. It necessitated institution of novel clinical pathways quickly across vastly different departments of obstetrics, with varied complements of providers. The guidance regarding screening and testing was ever-evolving in very short order. The escalating need for testing challenged the availability of equipment and supplies.

Overcoming these challenges to ensure that the processes were adopted across all sites taught us the following lessons:

(1) Co-create clinical pathways: medical leadership of NYC $\mathrm{H}+\mathrm{H}$ actively invited frequent check-ins together with the stakeholders in the Clinical Councils for women's health, pediatrics, and the laboratories to understand the needs of this seemingly healthier cohort of patients among the critically ill during the pandemic. These conversations uncovered the significant impact of the timing and the type of testing on management of these patients. The true collaboration across silos with open channels of communication gave voice to the advocates of the mother-baby dyad, highlighting the impact of universal testing on family-centered care, and allowing smoother implementation. 
(2) Adjust allocation of resources based on emerging needs: while standardization of universal testing is now recommended, the reality is that reagents and supplies for performing the test remain limited [11]. Laboratory Directors and Infection Prevention professionals were crucial in monitoring levels of these supplies and shifting resources based on individual facility needs. The rapid test (Cepheid rt-PCR) was in shorter supply than the Bioreference rt-PCR. The turnaround time for the Bioreference rt-PCR results still exceeded $48 \mathrm{~h}$ in most cases, which meant the testing of asymptomatic patients such as pregnant women was less meaningful in treatment decisions. An innovative plan was put into place to obtain BioReference rt-PCR at their last prenatal appointment - the last encounter prior to delivery. Cepheid rtPCR was reserved for patients with a shortened anticipated time to delivery. Upon implementation of universal testing, majority of the women admitted for delivery were tested using the Cepheid rt-PCR.

(3) Share clinical data in real time: clinical data for testing were shared in real time with comparisons across all facilities. The transparent communication of data uncovered location-specific barriers to implementation of universal testing. Real-time data sharing with late-adopting sites helped all perinatal-neonatal stakeholders advocate for the implementation of universal testing in obstetric patients.

The evolution of screening and testing for women admitted in labor at the largest U.S. public health system in order to guide clinical management and to stop the spread of SARS-CoV-2 is demonstrated in this paper. NYC $\mathrm{H}+\mathrm{H}$ was not alone in struggling to adapt to quickly changing guidance and data in reference to SARS-CoV-2. The prevalence of SARS-CoV-2 patients in our patients was significantly higher than earlier reports at the peak of the surge in pandemic in New York City [3]. This is likely a reflection of the patient population we serve as the safety net health system for the city. Overcoming the barriers to achieve successful transition from universal screening of patients' symptoms to universal testing of all patients admitted to labor and delivery units across our eleven acute care facilities required a great deal of coordination and collaboration. The work was complex and there were many obstacles impeding implementation of universal testing. These early lessons may help efficient adoption and successful implementation of universal testing in healthcare facilities facing a surge of SARS-CoV-2.

Acknowledgements We thank all members of the NYC $\mathrm{H}+\mathrm{H}$ Perinatal COVID Research Subcommittee: WW MD, KB MD, YM MD,
DW MD, Judith Chervenak MD, Daniel Lasser MD, Andrew Wiznia MD, Nichola Davis MD, Roopa Marcello MPH, Joseph Masci MD, and Ivan Hand MD. We also thank Hugo Theo for data extraction and Tianying $\mathrm{Li}$ (Mia) for data analyses.

\section{Compliance with ethical standards}

Conflict of interest The authors declare that they have no conflict of interest.

Publisher's note Springer Nature remains neutral with regard to jurisdictional claims in published maps and institutional affiliations.

\section{References}

1. Centers for Disease Control and Prevention. Considerations for inpatient obstetric healthcare settings. Centers for Disease Control and Prevention; 2020. https://www.cdc.gov/coronavirus/2019ncov/hcp/inpatient-obstetric-healthcare-guidance.html.

2. Breslin N, Baptiste C, Miller R, Fuchs K, Goffman D, GyamfiBannerman C, et al. Coronavirus disease 2019 in pregnancy: early lessons. Am J Obstet Gynecol MFM. 2020;2:100111.

3. Sutton D, Fuchs K, D'Alton M, Goffman D. Universal screening for SARS-CoV-2 in women admitted for delivery. N Engl J Med. 2020. https://doi.org/10.1056/NEJMc2009316.

4. Breslin N, Baptiste C, Gyamfi-Bannerman C, Miller R, Martinez $\mathrm{R}$, Bernstein $\mathrm{K}$, et al. COVID-19 infection among asymptomatic and symptomatic pregnant women: two weeks of confirmed presentations to an affiliated pair of New York City Hospitals. Am J Obstet Gynecol MFM. 2020. https://doi.org/10.1016/j.ajogmf. 2020.100118.

5. London V, McLaren R Jr, Atallah F, Cepeda C, McCalla S, Fisher $\mathrm{N}$, et al. The relationship between status at presentation and outcomes among pregnant women with COVID-19. Am J Perinatol. 2020. https://doi.org/10.1055/s-0040-1712164.

6. Gagliardi L, Danieli R, Suriano G, Vaccaro A, Tripodi G, Rusconi $\mathrm{F}$, et al. Universal SARS-CoV-2 testing of pregnant women admitted for delivery in two Italian regions. Am J Obstet Gynecol. 2020. https://doi.org/10.1016/j.ajog.2020.05.017.

7. Bianco A, Buckley AB, Overbey J, Smilen S, Wagner B, Dinglas $\mathrm{C}$, et al. Testing of patients and support persons for coronavirus disease 2019 (COVID-19) infection before scheduled deliveries. Obstet Gynecol. 2020. https://doi.org/10.1097/AOG. 0000000000003985.

8. Recommendations to the governor to reduce maternal mortality and racial disparities. New York State Taskforce on Maternal Mortality and Disparate Racial Outcomes; 2019. http://www.hea lth.ny.gov/community/adults/women/task_force_maternal_morta lity/docs/maternal_mortality_report.pdf.

9. Roche Diagnostics. Cobas ${ }^{\circ}$ SARS CoV-2 assay. Indianapolis, IN: Roche Diagnostics. https://www.roche.com/media/releases/medcor-2020-03-13.htm. Accessed 2020.

10. Food and Drug Administration. Xpert ${ }^{\oplus}$ Xpress SARS-CoV-2: instructions for use. Food and Drug Administration. https://www. fda.gov/media/136314/download. Accessed 2020.

11. American College of Obstetrics and Gynecology. Novel coronavirus 2019 (COVID-19). American College of Obstetrics and Gynecology. 2020. https://www.acog.org/clinical/clinicalguidance/practice-advisory/articles/2020/03/novel-coronavirus2019. 